\title{
SAFETY AND USEFULNESS OF COLONFIBROSCOPY IN THE VERY ELDERLY
}

\author{
Shu-Chen Wei, Jau-Min Wong, Ming-Jium ShIeH, Cheng-Yi Wang \\ Department of Internal Medicine, National Taiwan University Hospital, Taipei, Taiwan
}

\section{ABSTRACT}

The population of elderly people has increased in recent years. The importance of endoscopy as a diagnostic tool is indisputable. The feasibility and safety of upper gastrointestinal tract panendoscopy and sigmoidoscopy in elderly patients has been reported previously. However, there are still no reports about colonfibroscopy in the elderly, especially for the very elder patients (aged 80 years or above). This retrospective study reviewed the chart and endoscopic records of patients aged 80 or more who underwent colonfibroscopy examination at our hospital from January 1992 to December 1997. The basic clinical information, indications of endoscopy, the diagnostic results, and the followup conditions were all recorded and analyzed. A total of 240 colonfibroscopy examinations were performed in 189 patients. All the patients tolerated the procedure well. No complications were noted during and after the colonfibroscopy studies. The most frequent indication of colonfibroscopy was bloody stool and or positive stool occult blood test. In considering the final diagnosis, adenoma and or polyp, hemorrhoids, and colorectal cancer were the three most frequent etiologies in these patients. Only 17 examinations could not be properly completed due to poor colon preparation. Colonfibroscopy was a safe and useful diagnostic tool for the very elder patients. These very elderly patients had a high incidence of colorectal cancer, especially for those who had a past history of colorectal cancer (metachronous cancer). Those who had bloody stool and or positive stool occult blood test, bowel habit change or anemia, and who have a history of colorectal cancer should undergo the colonfibroscopy.

Biomed Eng Appl. Basis Comm, 2002 (February); 14: 31-34.

Key words: colonfibroscopy, very elderly.

\section{INTRODUCTION}

As the world population continues to age, the need for colonfibroscopy in the very elderly will increase. Many authors have stressed the safety and feasibility of colonfibroscopy for elderly patients with ailments related to the digestive tract [1-5]. Many previous reports have discussed the results of upper gastrointestinal tract panendoscopy and sigmoidoscopy in

Received: November 7, 2001; accepted: February 3, 2002. Correspondence: Cheng-Yi Wang, Professor Department of Internal Medicine, National Taiwan University Hospital, No. 7 Chung-Shan South Road, Taipei, Taiwan. elderly patients. However, there are still no reports which have restricted their study of colonfibroscopy to the very elderly patients (aged 80 years or above). The aim of this study was to explore the safety and usefulness of colonfibroscopy in the very elderly.

\section{METHODS}

This retrospective study was conducted to explore the diagnostic role and safety of colonfibroscopy for the very elder patients (aged 80 years or above). We reviewed the medical charts and endoscopic records of all very elderly patients who had received colonfibroscopic studies at National Taiwan University Hospital from January 1992 to December 1997. The indications 
for colonfibroscopy, diagnostic results, endoscopic findings, histology results, follow-up periods and patients' survival status, and demographic information were recorded and analyzed. Patients were prepared with a low fiber diet for 3 days as possible. Bowel cleaning was then performed with magnesium citrate oral solution and bisacodyl one night before the examination. Intramuscular meperidine hydroxy chloride was used for sedation and hyoscine butylbromide for anti-spasmodic effect of the bowel were routinely given before the procedure, except for patients' whose clinical condition could not tolerate the medications.

\section{RESULTS}

From January 1992 to December 1997, a total of 240 colonfibroscopy examinations were performed in 189 patients aged 80 or above at National Taiwan University Hospital. The National Taiwan University Hospital is a tertiary care center at Taipei in Taiwan. There were 72 women and 117 men with a mean age of 81.6 years (range, 80.0 to 87.6). The postexamination follow-up period ranged from one month to 6.5 years (mean 2.2 years). Among these 240 examinations, $140(58.3 \%)$ were done in the out-patientdepartment, $89(37.1 \%)$ in the general ward, three $(1.3 \%)$ in the emergency room, four $(1.7 \%)$ in the intensive care unit, and four $(1.7 \%)$ in the general checkup unit. Preparative medications for colonfibroscopy included meperidine hydroxychloride $92.1 \%(221 / 240)$, and hyoscine butylbromide $97.1 \%$ (233/240). All patients tolerated the procedure well. No complications were noted during and after the colonfibroscopy studies. Thirty-eight patients died during the follow-up period. One died of gastrointestinal tract bleeding, 22 died of infection (pneumonia and/or urinary tract infection), 11 died of heart failure and arrhythmia, two died of colon cancer with complications and two died of sarcoma with complications.

The indications for colonfibroscopy in these patients are listed in Table 1. The most frequent indications were bloody stool and/or positive stool occult blood test, adenoma and/or polyp follow-up, and bowel habit change, respectively. The three most frequent etiologies in these patients were adenoma and/or polyp, hemorrhoids, and colorectal cancer. The diagnostic results are summarized in Table 2 . Thirteen patients had colorectal cancer as well as adenoma and/or polyp. Only 17 examinations could not be completed due to poor colon preparation. The locations of the lesions identified by colonfibroscopy are shown in Table 3. The most frequent indication for colonfibroscopy was bloody stool and/or positive stool occult blood test. The final diagnoses of these patients are summarized in Table 4. Adenoma and/or polyp were the most common diagnosis. The indications for performing colonfibroscopy in patients with a final diagnosis of colorectal cancer are shown in Table 5 .

\section{DISCUSSION}

As a diagnostic procedure, colonfibroscopy is relatively more difficult than other type of endoscopy and might carry a higher complication rate also. The

Table 1. The Indications for colonfibroscopy in very elderly patients.

\begin{tabular}{lll}
\hline Indication & Frequency & Percent \\
\hline Bloody stool and/or Positive stool occult blood test & 66 & 27.5 \\
Adenoma and/or polyp, F/U & 61 & 25.4 \\
Bowel habit change & 35 & 14.6 \\
Suspicious lesion by abdominal sonography or barium enema & 27 & 11.3 \\
Colon cancer s/p operation, Follow-up & 23 & 9.6 \\
Anemia & 9 & 3.8 \\
Abdominal pain & 7 & 2.9 \\
Physical examination & 4 & 1.7 \\
Increase of carcinoembryonic antigen (CEA) & 4 & 1.7 \\
Searching for primary site of metastatic cancer & 2 & 0.8 \\
Carcinoid tumor, Follow-up & 1 & 0.4 \\
Ileus & 1 & 0.4 \\
\hline Total & 240 & 100 \\
\hline
\end{tabular}


Table 2. Colonfibroscopic diagnosis in very elderly patients.

\begin{tabular}{ll}
\hline Diagnosis & Frequency \\
\hline Polyp/adenoma & 97 \\
Hemorrhoid & 54 \\
Cancer & 40 \\
Colitis & 33 \\
$\quad$ Nonspecific & 25 \\
CDAD & 2 \\
Ulcer & 2 \\
Ischemia & 2 \\
Radiation & \multicolumn{2}{c}{25} \\
Ameba & \multicolumn{1}{c}{1} \\
Negative & 23 \\
Diverticulum & 18 \\
Poor preparation & 17 \\
Vascular lesion (Angiodysplasia/Telangiectasia) & 5 \\
Gastrointestinal tract bleeding, source uncertain & 5 \\
\hline
\end{tabular}

Table 3 . The locations of lesions identified by colonfibroscopy in very elderly patients.

\begin{tabular}{lll}
\hline Locations & Frequency & Percent \\
\hline Anus/Rectum & 57 & 29.2 \\
Sigmoid colon & 35 & 17.9 \\
Descending colon & 9 & 4.6 \\
Transverse colon & 7 & 3.6 \\
Ascending colon & 16 & 8.2 \\
Cecum & 5 & 2.6 \\
Multiple, distal colon & 28 & 14.4 \\
Multiple, proximal colon & 10 & 5.1 \\
Multiple, distal and proximal colon & 27 & 14.4 \\
\hline Total & 195 & 100 \\
\hline
\end{tabular}

safety of colonfibroscopy and the colonic disease patterns in the very elderly remain unclear. In this study, there were no complications during and after 240 colonfibroscopy procedures. All of the 198 patients tolerated the procedure well. Thus, colonfibroscopy seems to be a safe procedure for the very elderly.

Flexible sigmoidoscopy is superior to rigid sigmoidoscopy, as it allows examination of longer lengths of bowel and has a five- to sixfold greater diagnostic yicld than rigid sigmoidoscopy [3]. The relative superiority between flexible sigmoidoscopy and colonfibroscopy is less clear. According to the locations of the identified lesions by colonfibroscopy in our patients, $33.9 \%$ would have been missed if we had only performed the sigmoidoscopy. By using the same bowel preparation and pre-procedure medications, we suggest that colonfibroscopy is a better diagnostic tool than sigmoidoscopy which has a similar complication rate but obtains a higher diagnostic yield.

Double-contrast enema is another option for studying the colon. In a previous study, the rate of successful completion was $94.9 \%(113 / 119)$ in patients in the eighth decade, and $90 \%(81 / 90)$ in those 80 and over. Most unsuccessful double contrast enemas were due to the patient's inability to retain barium or the enema tip itself, and occurred mostly in the nonambulatory patients [6]. In our colonfibroscopic studies, $7.1 \%(17 / 240)$ of failures were due to poor preparation and $2.1 \%(5 / 240)$ were due to bleeding from a source which could not be clearly identified on colonfibroscopy. Overall, the success rate was still higher than that reported for double contrast enema. In this study, poor bowel preparation was also mostly seen in patients with disability. Endoscopy can identify minor lesions such as vascular lesions and inflammatory change and allows for biopsy for histology studies. We recommend colonfibroscopy for elderly patients instead of sigmoidoscopy or double contrast enema.

In this series, the most frequent indication for colonfibroscopy was bloody stool and/or positive stool occult blood test. The most prevalent diagnoses were adenoma and/or polyp followed by colitis, hemorrhoid, colorectal cancer for patients with this indication (Table 4$)$. A relatively high incidence $16.7 \%(40 / 240)$ of 
Table 4. The final diagnosis among very elderly patients with the colonfibroscopic indication of bloody stool and/or positive stool occult blood test

\begin{tabular}{ll}
\hline Diagnosis & Frequency \\
\hline Adenoma and/or polyp & 21 \\
Colitis & 14 \\
Hemorrhoid & 10 \\
Colorectal cancer & 9 \\
Diverticulum & 7 \\
Poor preparation & 5 \\
Gastrointestinal tract bleeding, source uncertain & 5 \\
Negative & 4 \\
Angiodysplasia/ Telangiectasia & 2 \\
\hline
\end{tabular}

Table 5. Indications for colonfibroscopy in very elderly patients with a final diagnosis of colorectal cancer

\begin{tabular}{lll}
\hline Indication & Frequency & Percent (\%) \\
\hline Bloody stool and/or positive stool occult blood test & 9 & 22.5 \\
Suspicious lesion by abdominal sonography or barium enema & 9 & 22.5 \\
Colorectal cancer s/p operation & 8 & 20 \\
Bowel habit change & 6 & 15 \\
Anemia & 4 & 10 \\
Adenoma/polyp & 2 & 5 \\
Abdominal pain & 1 & 2.5 \\
Ileus & 1 & 2.5 \\
\hline Total & 40 & 100 \\
\hline
\end{tabular}

colorectal cancer was found in this series. It is generally agreed that the incidence of cancer increases with age $[7,8]$. In this series, the three most frequent presentations of colorectal cancer were bloody stool and/or positive stool occult blood test, bowel habit change and anemia (Table 5). Therefore, the occurrence of these symptoms in the very elderly should lead to suspicion of colorectal cancer and prompt colonfibroscopy is highly recommended. Among the follow-up patients with a history of colorectal cancer, a very high percentage $(34.7 \%, 8 / 23)$ of metachronous colorectal cancer was found on colonfibroscopy. The necessity of regular colonfibroscopic follow-up for patients with colorectal cancer should not be overlooked.

In summary, in this series, colonfibroscopy was a safe and useful diagnostic tool for very elderly patients. Bloody stool and/or positive stool occult blood test was the most frequent indication for colonfibroscopy, and adenoma and/or polyp was the most common diagnosis. These very elderly patients had a high incidence of colorectal cancer. Therefore, we conclude that very elderly patients with bloody stool and/or positive stool occult blood test, bowel habit change or anemia, and those with a history of colorectal cancer should undergo the colonfibroscopy examination.

\section{REFERENCES}

1. Jacobson WZ, Levy A. Endoscopy of upper gastrointestinal tract is feasible and safe in elderly patients. Geriatrics. 1977; 1: 80-83.

2. Lee YC, Tam HC. Upper gastrointestinal endoscopy for Chinese elderly in an acute geriatric unit: a review. Age Ageing. 1984; 13: 285-290.

3. Helzberg JH, Mccallum RW. Flexible sigmoidoscopy: safety and usefulness in the geriatric patient. Geriatrics. 1985; 40: 105-108.

4. Brussaard CC, Vandewoude MFJ. A prospective analysis of elective upper gastro- intestinal endoscopy in the elderly. Gastrointest Endosc. 1988; 34:118-121.

5. Holt RW, Wherry DC. Why flexible fiberoptic sigmoidoscopy is important in the geriatric patient. Geriatrics. 1979; 5: 85-88.

6. Wolf EL, Frager D, Beneventano TC. Feasibility of double-contrast barium enema in the elderly. $A J R$ Am J Roentgenol. 1985; 145: 47-8.

7. Bolin S, Nilsson E, Sjodahl R. Carcinoma of colon and rectum. Growth rate. Ann Surg. 1983; 198:151.

8. Minaker K, Rowe J. Gastrointestinal system. In: Health and Disease in old age. Rowe J., Besdine R. eds. Boston: Little Brown and Company; 1982. 